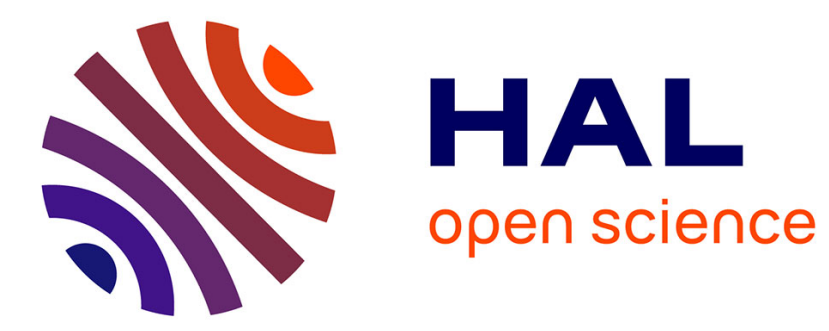

\title{
SNR Improvement by Subharmonic Extraction with Hammerstein Models for Microbubble Signals
}

Sébastien Ménigot, Emma Kanbar, Ayache Bouakaz, Jean-Marc Girault

\section{To cite this version:}

Sébastien Ménigot, Emma Kanbar, Ayache Bouakaz, Jean-Marc Girault. SNR Improvement by Subharmonic Extraction with Hammerstein Models for Microbubble Signals. IEEE International Ultrasonic Symposium 2016, IEEE UFFC, Sep 2016, Tours, France. 10.1109/ULTSYM.2016.7728740 . hal-01371261

\section{HAL Id: hal-01371261 \\ https://hal.science/hal-01371261}

Submitted on 24 Nov 2016

HAL is a multi-disciplinary open access archive for the deposit and dissemination of scientific research documents, whether they are published or not. The documents may come from teaching and research institutions in France or abroad, or from public or private research centers.
L'archive ouverte pluridisciplinaire HAL, est destinée au dépôt et à la diffusion de documents scientifiques de niveau recherche, publiés ou non, émanant des établissements d'enseignement et de recherche français ou étrangers, des laboratoires publics ou privés. 


\title{
SNR Improvement by Subharmonic Extraction with Hammerstein Models for Microbubble Signals
}

\author{
Sébastien Ménigot*†, Emma Kanbar*‡, Ayache Bouakaz ${ }^{* \S}$ and Jean-Marc Girault* \\ *Université François-Rabelais de Tours, Inserm, Imagerie et Cerveau UMR U930, Tours, France \\ $\dagger$ sebastien.menigot@univ-tours.fr, corresponding author \\ $\ddagger$ emma.kanbar@etu.univ-tours.fr \\ §ayache.bouakaz@univ-tours.fr \\ 9jean-marc.girault@univ-tours.fr
}

\begin{abstract}
Nowadays, ultrasound imaging contrast has enhanced the contrast thanks to the nonlinear behaviour of microbubbles. Unfortunately, this contrast improvement is compromised by the nonlinear acoustic propagation in tissue. Fortunately, microbubbles can also oscillate with a $f_{0} / 2$-subharmonic frequency. Therefore, as there is no subharmonic in the tissue backscattering, the subharmonic imaging could increase the contrast highly. However, the subharmonic extraction can be more or less empirical by using standard filters. The aim of this study is thus to propose an optimal extraction of subharmonics. As Hammerstein model fits well the nonlinear systems with harmonics, we propose two solutions based on a combination of such a model to fit nonlinear systems with sub and ultra-harmonics. Experiments are carried out by transmitting ultrasound wave at $10 \mathrm{MHz}$ to a water-diluted solution of Sonovue. The nonlinear microbubble responses were measured by a $5 \mathrm{MHz}$ transducer. Results showed that the error between the experimental signal and the modeling signal was less than $-18 \mathrm{~dB}$. Moreover, the bias was always less than -15 $\mathrm{dB}$. Finally, the signal-to-noise ratio could be increase by $4 \mathrm{~dB}$ in comparison with a standard filtering. Thanks to the modified Hammerstein model, it was possible to set the extraction optimally adjusted to the subharmonic components. No manual analysis of the signals was required. ${ }^{1}$
\end{abstract}

\section{INTRODUCTION}

Diagnostic ultrasound imaging have been brought about a revolution by ultrasound contrast agents (UCA) for about twenty years [1]. The microbubbles constituting UCA have improved the contrast by taking into account their high nonlinear behavior [2]. Therefore, ultrasound contrast imaging traditionally consists in transmitting a wave at the frequency $f_{0}$ and in receiving the echoes at a integer multiple of the transmit frequency such $2 f_{0}$ or $3 f_{0}$. This backscattered harmonic component are extracted by frequency filtering [3]. However, the filter setting can be difficult by taking into account the transducer bandwidth. Moreover, many methods using a combination of pulses [4] have been developed to remove this frequency filtering with the prize of reducing the frame rate. To overcome this problem, a nonlinear mean average model (NMA) can be applied to optimally extract the harmonic components [5]. Unfortunately, these attempts have been reduced, because the contrast is limited by the nonlinear acoustic propagation of the tissue.

Fortunately, the nonlinear behavior of UCA can also generate sub and ultraharmonic components at $f_{0} / 2$ and $3 f_{0} / 2$ respectively, with particular acoustic [6] and physical [7] conditions. This property could highly increase the contrast, because no subharmonic components are generated by tissues [8]. The problem is thus to adjust the previous method to extract subharmonic components. A Volterra modelisation has been developed by changing from the single input system to a multiple input system [9], [10]. Despite a low error between the microbubble signal and the model, no subharmonic extraction was possible. However, an easier decomposition was proposed by using a combination of Hammerstein models [11]. It has enables the subharmonic extraction. Nevertheless, this method was only applied on simulated microbubble signals.

In this paper, we propose to apply two combinations of Hammerstein models on real microbubble signals. This technique required only the transmit signal and the order of subharmonic components. No assumptions of the filtering bandwidth is required.

\section{Subharmonic EXtraction}

The subharmonic extraction which is proposed here is based on a Hammerstein model. Such Hammerstein model is usually decomposed by parallel subsystems as a polynomial function and a linear filter. As an illustration, examples of spectra of input and output signals are presented in Fig. 1. The two first spectra show the input and the output where the system works nonlinearly with subharmonic components (in green). The standard polynomial Hammerstein model can model only the integer

${ }^{1}$ DOI : 10.1109/ULTSYM.2016.7728740

(c) 2016 IEEE. Reprinted, with permission, from Sébastien Ménigot, Emma Kanbar, Ayache Bouakaz and Jean-Marc Girault, SNR improvement by subharmonic extraction with Hammerstein models for microbubble signals, 2016 IEEE International Ultrasonics Symposium (IUS), 2016. This material is posted here with permission of the IEEE. Such permission of the IEEE does not in any way imply IEEE endorsement of any of the Université François Rabelais de Tours' products or services. Internal or personal use of this material is permitted. However, permission to reprint/republish this material for advertising or promotional purposes or for creating new collective works for resale or redistribution must be obtained from the IEEE by writing to pubs-permissions@ieee.org. 
harmonic components, as in the third spectrum. However, in order to model the subharmonics at the frequency $f_{0} / 2$, either the input (switch in position 1) or the output (switch in position 2) should be modulated. By combining both, it is possible to model the whole spectrum.

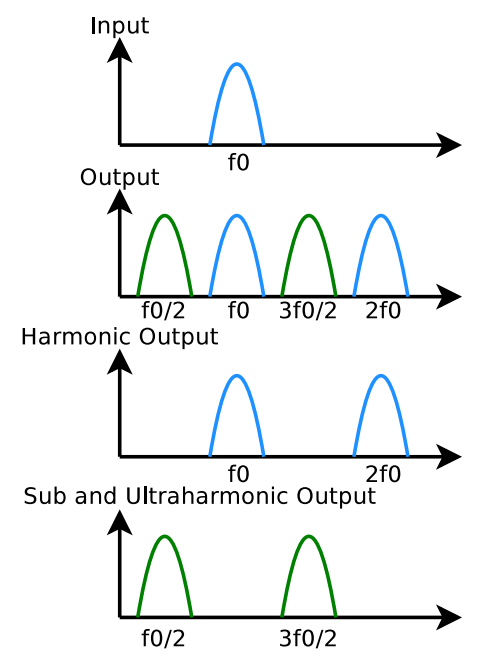

Fig. 1. Scheme of spectra of input and output signals.

\section{A. Hammerstein Model}

As previoulsy mentioned, the proposed method is slightly different than the one proposed in [11]. The difference lies in replacement of the polynomial function by modulation functions. Note that this modification is possible, since the continuous components modelled by the even order are deleted because of the transducer filtering. Therefore, the model (Fig. 2) consider all the components as harmonics, either by demodulated the input or by modulated the ouput.

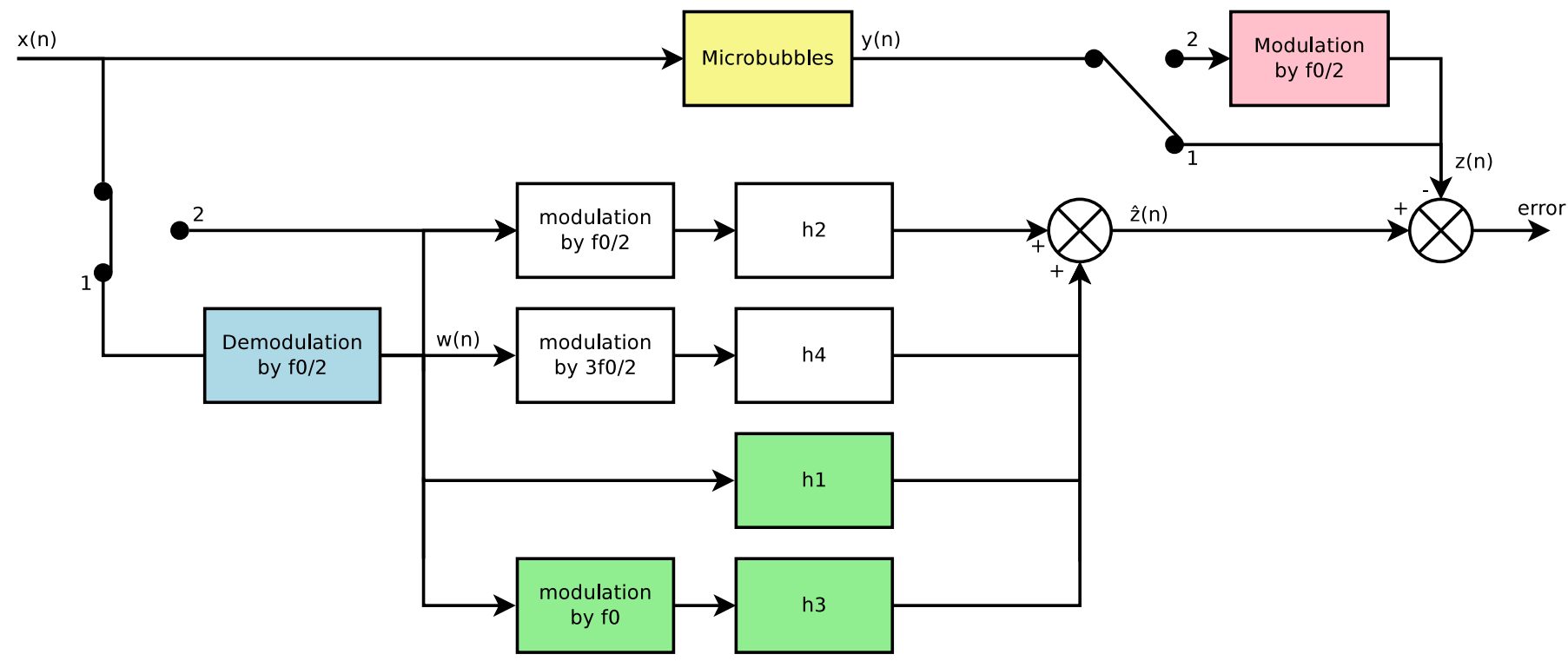

Fig. 2. Block diagram of second order Hammerstein model adapted for subharmonic components. The blue and pink functions are the two solutions to model the subharmonics. The sum of green functions enables the subharmonic extraction.

Therefore, the signal modelling $\hat{z}(n)$ of the microbubble signal $y(n)$ can be written as:

$$
\hat{z}(n)=\sum_{p=1}^{2 P} \sum_{m=1}^{M} h_{p}(m) w(n-m) \cdot C_{p}(n),
$$


where $n$ is the discrete time, $M$ the memory of the Hammerstein model, $P$ its order, $w(n)$ the input model and $C_{p}(n)=$ $\cos \left(2 \pi \frac{(p-1) \frac{f_{0}}{2} n}{F_{s}}\right)$ with $F_{s}$ the sampling frequency. Note that the order $P$ is limited at 2 , since the transducer bandwidth removes the high order harmonics. Therefore, the Hammerstein modelling is solved by finding the linear filter coefficients $h_{p}$.

This problem can be written with an algebraic formula, such as :

$$
\hat{\mathbf{z}}=\mathbf{W}^{T} \mathbf{h},
$$

where $\hat{\mathbf{z}}=[z(M+1), \ldots, z(N)]^{T}$ with $N$ the sample quantity and ${ }^{T}$ the symbol of the vector transposition, $\mathbf{h}$ the vector of coefficients

$$
\mathbf{h}=\left[h_{1}(1), \ldots, h_{1}(M), h_{2}(1), \ldots, h_{4}(M),\right]^{T},
$$

and $\mathbf{W}=\left[\mathbf{w}_{1} \mathbf{w}_{2} \ldots \mathbf{w}_{4}\right]$ with

$$
\mathbf{w}_{p}=\left(\begin{array}{llll}
v_{p}(M) & v_{p}(M+1) & \ldots & v_{p}(N) \\
v_{p}(M+1) & v_{p}(M+2) & & 0 \\
\vdots & & . \cdot & \\
v_{p}(N) & 0 & & 0
\end{array}\right)
$$

and $v_{p}(n)=w(n) C_{p}(n)$. Finding a solution minimizing the error between the signal modelling $\hat{z}(n)$ and the microbubble signal $y(n)$ can be obtained with a pseudo-inversion, such as:

$$
\mathbf{h}=\left(\mathbf{W}^{T} \mathbf{W}\right)^{-1} \mathbf{W}^{T} \mathbf{z} .
$$

\section{B. Input and Output Model}

As demonstrated bellow, the sub and ultraharmonic modelling is possible thanks to a combination of two Hammerstein models reported in Fig. 2.To do so, the lower frequency components in the output should be equal to the transmit frequency $f_{0}$. Without modifying either the input or the output of the second Hammerstein structure, the subharmonics components is impossible. Therefore two solutions are possible. In the first solution (blue function on Fig. 2), the input is demodulated by $f 0 / 2$ such as:

$$
\left\{\begin{array}{l}
w(n)=x(n) \cdot \cos \left(2 \pi \frac{\frac{f_{0}}{2} n}{F_{s}}\right) ; \\
z(n)=y(n) .
\end{array}\right.
$$

In the the second solution (pink function on Fig. 2), the output is modulated by $f 0 / 2$ such as:

$$
\left\{\begin{array}{l}
w(n)=x(n) ; \\
z(n)=y(n) \cdot \cos \left(2 \pi \frac{\frac{f_{0}}{2} n}{F_{s}}\right) .
\end{array}\right.
$$

Note that for this second solution, the modelling signal has to be demodulated by $f_{0} / 2$.

\section{Subharmonic Reconstruction}

Finally, the subharmonics extraction is based on the odd coefficients $\mathbf{h}_{\text {sub }}=\left[h_{1}(1), \ldots, h_{1}(M), h_{3}(1), \ldots, h_{3}(M)\right]^{T}$ (green functions on Fig. 2). The subharmonic signal $\mathbf{z}_{\text {sub }}$ can be modeled as:

$$
\mathbf{z}_{\text {sub }}=\mathbf{w}_{1}^{T} \mathbf{h}_{\text {sub }} .
$$

Note that a Matlab program of this solution is available on RunMyCode.org (http://www.runmycode.org/coder/view/2395).

\section{EXPERIMENTAL SETUP}

Experiments are carried out by using two transducers which were placed perpendicularly to avoid direct transmission (Fig. 3). The excitation signal was transmitted through a GPIB port (National Instruments, Austin, TX) to an arbitrary function generator (33220A, Agilent, Palo Alto, CA). The signal was then amplified using a power amplifier (Amplifier Research 150A100B, Souderton, PA) and transmitted to a $10 \mathrm{MHz}$ PZT single element (V311, Olympus Panametrics, Waltham MA, USA). Responses of a diluted solution of Sonovue ${ }^{\mathrm{TM}}$ microbubbles (Bracco Research, Geneva, Switzerland) were measured by a $5 \mathrm{MHz}$ PZT single element transducer. Measured echoes were amplified by $30 \mathrm{~dB}$ (Pulser-Receivers 5072R, Olympus Panametrics, Waltham, MA, USA) and then visualized on a digital oscilloscope (Tektronix, Beaverton, OR). Signals were transferred to a personal computer through a GPIB port for further analysis. Finally, the subharmonic components are extracted with Matlab® (Mathworks, Natick, MA, USA). 


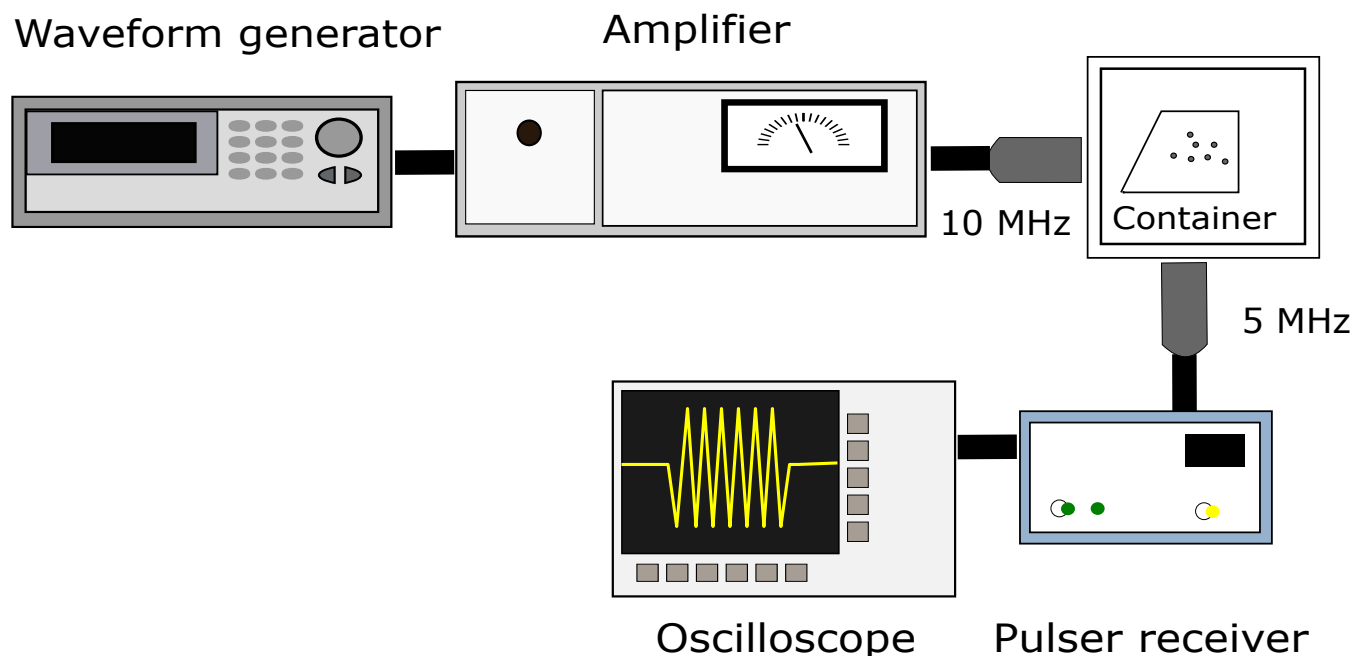

Fig. 3. Experimental setup.

\section{RESUlts}

The two solutions were applied on a microbubble signal: method 1 with input demodulation and method 2 with output modulation. Moreover, to model the signal on the full duration, the memory $M$ had to be equal to the sample number, as here 5000 .

Fig. 4 shows the spectra of the system input and the output (microbubble, method 1 and method 2). The input demodulation is depicted on Fig. 4b and the output modulation on Fig. 4c. Both modellings are superimposed in red line. These modellings are visually identical to the microbubble spectrum. Therefore, the Hammerstein structures could model correctly the microbubble signals including subharmonics.

Fig. 5a shows the same results in a time view. The error were low. Moreover, Figs. 5b-c show the subharmonic extraction in a time and a frequency representation. The subharmonics were extracted and separated from other harmonic components. They were correctly modelled by comparing the subharmonics in the microubble spectrum. As an illustration, the subharmonic extractions were compared with a standard filtering. Note that this filter is a Butterworth filter centred at $5 \mathrm{Mhz}$ with a bandwidth of $40 \%$ at $-3 \mathrm{~dB}$. The main subharmonic component was identical. However, the bandwidths were sightly higher with the standard filtering, and the standard filtering could extend the overlap with the fundamental components.

Fig. 6 shows the quantitative comparison between the different method. Fig. 6a shows the signal-to-ratio evaluated on the subharmonics for the method 1, the method 2 and the standard frequency filtering. Fig. $6 \mathrm{~b}$ and c repectively show the root mean square error and the bias between the Hammerstein model and the microbubble signal (on all the components). Both Hammerstein methods had similar performances. The error between the experimental signal and the modelling signal was less than $-35 \mathrm{~dB}$. This error was slightly inferior by using a demodulation of the output signal. Moreover, the bias was always less than $-50 \mathrm{~dB}$. Finally, the signal-to-noise ratio (SNR) could be increase by $4 \mathrm{~dB}$ in comparison with a standard filtering.

\section{Discussions AND CONCLUSION}

Thanks to the Hammerstein models, it was possible to set the extraction optimally adjusted to the subharmonic components. The method made possible as well as the subharmonics than the ultraharmonics in the same structure. No manual analysis of the signals was required. Only the knowledge of the center frequency of the input signal was required. This information is usually well-known by experimenter.

Moreover, the Hammerstein model minimized the error between the modelling and the microbubble signal. This optimization made possible to increase the SNR until $4 \mathrm{~dB}$. These performances were sightly better by using a output modulation. As the model is written from the input signal, avoiding the modification on the input signal may reduce the error.

Finally, in comparison with the previous study, the memory was higher because of the signal duration. Even if the time computation could increase, it remained reasonable.

To conclude, the subharmonic extraction from Hammerstein model seems to be a simple method to increase the SNR in the subharmonic signal. Ultrasound contrast imaging could benefit to this improvement. Moreover, this subharmonic extraction could be used in the studies of subharmonic microbubble behaviour. 
(a)



(b)

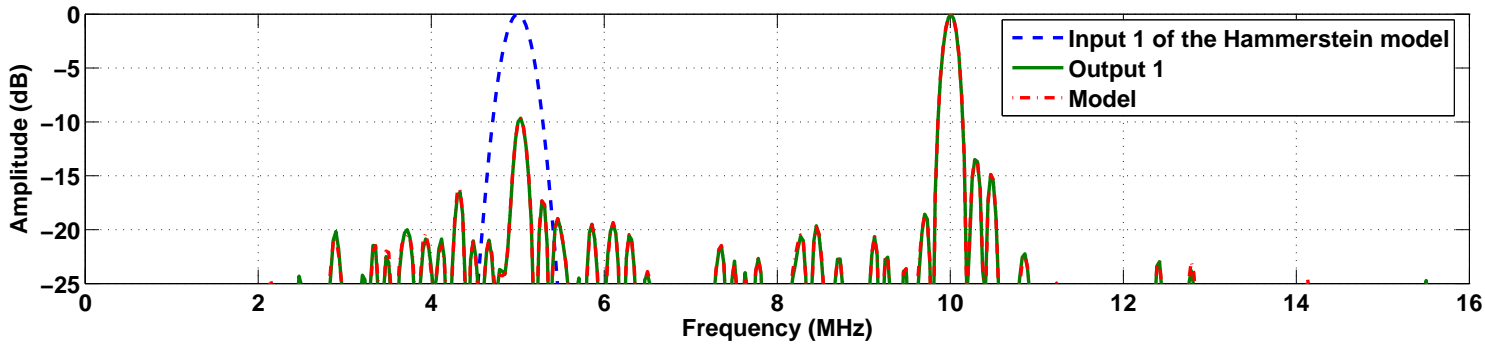

(c)

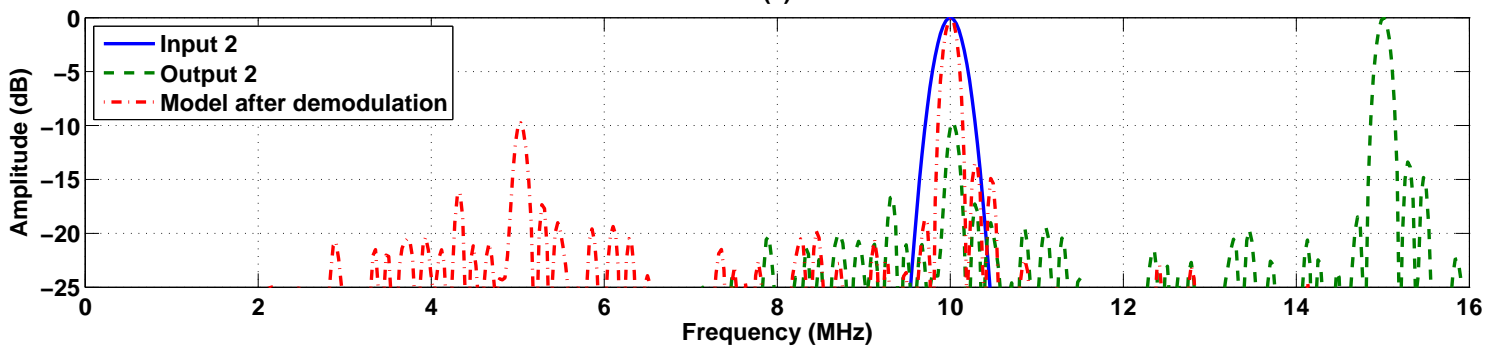

Fig. 4. (a) Spectra of microbubble excitation and of the microbubble backscattering. (b) Spectra of input, output and model for the method 1. (b) Spectra of input, output and model for the method 2. Note that method 1 included an input demodulation and method 2 an output modulation.

\section{REFERENCES}

[1] B. B. Goldberg, J.-B. Liu, and F. Forsberg, "Ultrasound contrast agents: A review," Ultrasound in Medicine and Biology, vol. 20, no. 4, pp. 319-333, 1994.

[2] N. de Jong, A. Bouakaz, and P. Frinking, "Harmonic imaging for ultrasound contrast agents," in Proceeding IEEE Ultrasonic Symposium, San Juan, Puerto Rico, Oct. 2000, pp. 1869-1876.

[3] P. N. Burns, "Instrumentation for contrast echocardiography," Echocardiography-A Journal Of Cardiovascular Ultrasound And Allied Techniques, vol. 19, no. 3, pp. 241-258, Apr. 2002.

[4] F. Lin, C. Cachard, F. Varray, and O. Basset, "Generalization of multipulse transmission techniques for ultrasound imaging," Ultrasonic Imaging, vol. 37, no. 4, pp. 294-311, Oct. 2015.

[5] P. Phukpattaranont and E. S. Ebbini, "Post-beamforming second-order volterra filter for pulse-echo ultrasonic imaging," IEEE Transactions on Ultrasonics Ferroelectrics and Frequency Control, vol. 50, no. 8, pp. 987-1001, Aug. 2003.

[6] F. Forsberg, W. T. Shi, and B. B. Goldberg, "Subharmonic imaging of contrast agents," Ultrasonics, vol. 38, no. 1-8, pp. 93-98, Mar. 2000.

[7] E. Kanbar, D. Fouan, C. A. Sennoga, and A. Bouakaz, "Impact of gas composition on subharmonic emission from phospholipid contrast agents," in The European symposium on Ultrasound Contrast Imaging, Rotterdam, The Netherlands, Jan. 2016.

[8] P. M. Shankar, P. Dala Krishna, and V. L. Newhouse, "Advantages of subharmonic over second harmonic backscatter for contrast-to-tissue echo enhancement." Ultrasound in Medicine and Biology, vol. 24, no. 3, pp. 395-399, Mar 1998.

[9] O. M. Boaghe and S. A. Billings, "Subharmonic oscillation modeling and miso volterra series," IEEE Transactions on Circuits and Systems I: Fundamental Theory and Applications, vol. 50, no. 7, pp. 877-884, 2003.

[10] F. Sbeity, J. Ménigot, S.and Charara, and J.-M. Girault, "A general framework for modeling sub- and ultraharmonics of ultrasound contrast agent signals with miso volterra series," Computational and Mathematical Methods in Medicine, vol. 2013, p. 9, 2013.

[11] F. Sbeity, S. Ménigot, J. Charara, and J.-M. Girault, "Contrast improvement in sub- and ultraharmonic ultrasound contrast imaging by combining several hammerstein models," International Journal of Biomedical Imaging, vol. 2013, p. 9, Sep. 2013. 
(a)
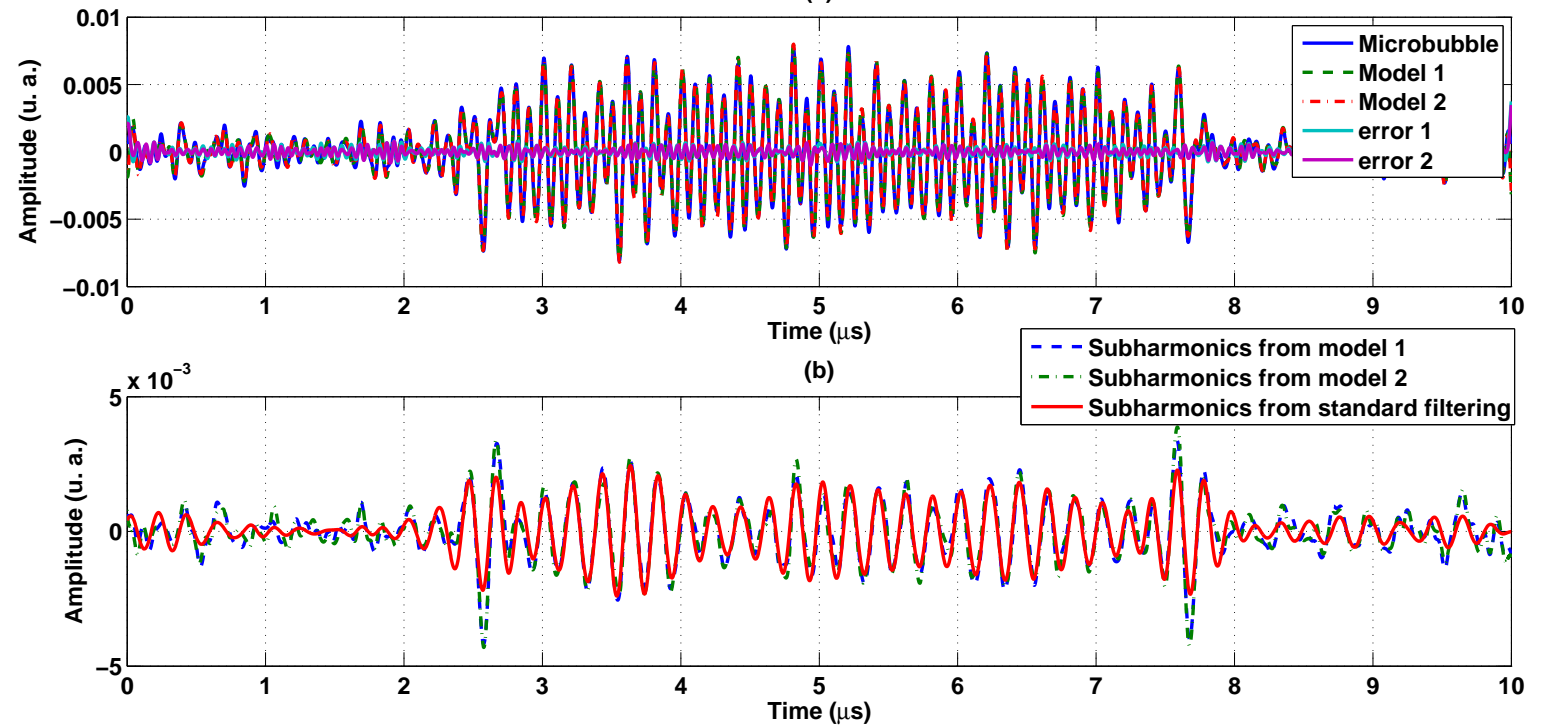

(c)

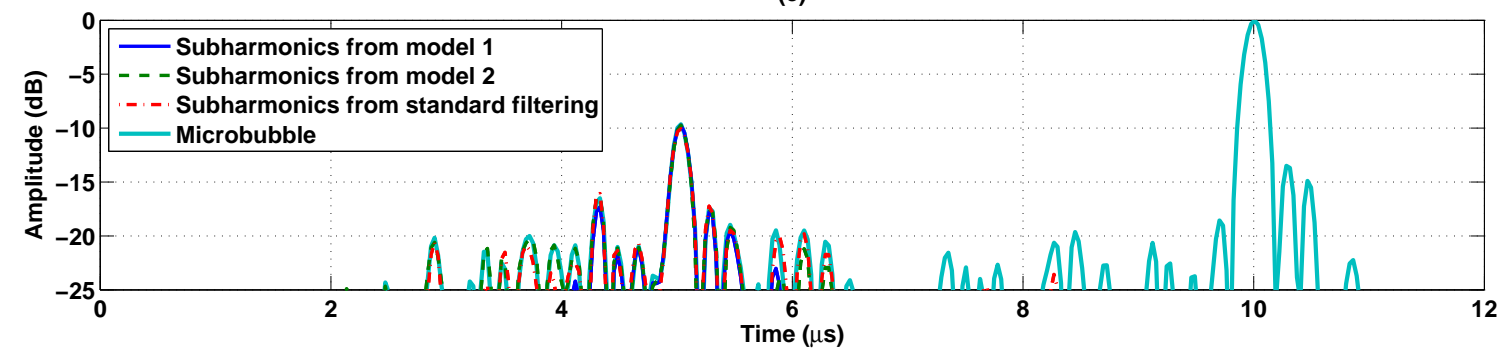

Fig. 5. (a) Microbuble signal, its modellings from method 1 and method 2 and the respective error between them. (b) Subharmonics signal from method 1 and method 2. (c) Spectra of subharmonics signal from method 1 and method 2. Note that method 1 included an input demodulation and method 2 an output modulation. 

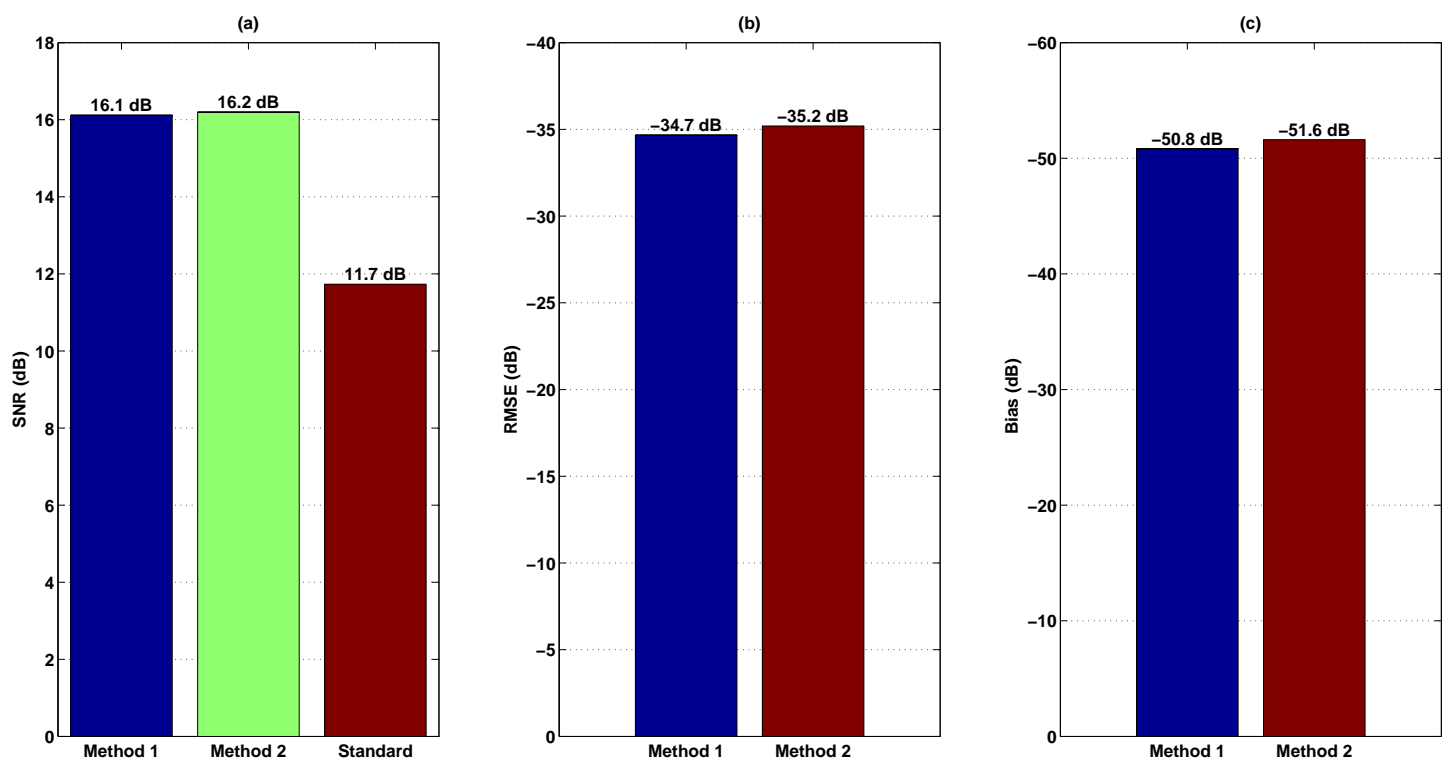

Fig. 6. (a) Signal-to-noise ratio with the method 1, the method 2 and the standard filtering. (b) Root mean square error for the method 1 and the method 2. (c) Bias for the method 1 and the method 2. Note that method 1 included an input demodulation and method 2 an output modulation. 